\title{
MEG gamma band activity in schizophrenia patients and healthy subjects in a mental arithmetic task and at rest
}

\author{
Johanna Kissler ${ }^{\mathrm{a}, *}$, Matthias M. Müller ${ }^{\mathrm{b}}$, Thorsten Fehr ${ }^{\mathrm{a}}$, Brigitte Rockstroh ${ }^{\mathrm{a}}$, Thomas Elbert ${ }^{\mathrm{a}}$ \\ ${ }^{a}$ Fachgruppe Psychologie, Universität Konstanz, Konstanz, Germany \\ ${ }^{\mathrm{b}}$ Cognitive Neuroscience and Neuropsychology, Department of Psychology, University of Liverpool, Liverpool, UK
}

Accepted 18 July 2000

\begin{abstract}
Objectives: High frequency oscillations have been suggested as a correlate of cognitive processes and have recently also been implicated in aberrant forms of information processing. The present study investigated whether magnetoencephalographic (MEG) gamma band activity (20-71 Hz) can serve as an index of cognitive processes in the absence of external stimulation and to what extent gamma activity differs between healthy people and schizophrenia patients.

Methods: The amount and topography of MEG power in the gamma band range was examined in 15 schizophrenia patients and 15 healthy comparison subjects while performing a complex mental arithmetic task and at rest.

Results: In healthy subjects a left frontal and left fronto-temporal increase in gamma power was observed during mental arithmetic. Schizophrenia patients either failed to display such a task effect $(30-45 \mathrm{~Hz})$ or had reversed lateralization with enhanced activity over right frontal and right fronto-temporal regions under cognitive demands $(45-71 \mathrm{~Hz})$. In the frequency band from 60 to $71 \mathrm{~Hz}$ patients showed less gamma at fronto-temporal, posterio-temporal and occipital sites irrespective of the task.

Conclusions: These results indicate, first, that gamma topography can index cognitive activation in a very complex and purely internal task. Second, groups differed in the pattern of activation during the task, a result which may be consistent with working memory dysfunction in schizophrenia. Third, the general topographic difference between healthy subjects and patients is in line with the notion of abnormalities in the thalamocortical circuit in schizophrenia. (C) 2000 Elsevier Science Ireland Ltd. All rights reserved.
\end{abstract}

Keywords: MEG; Gamma band; Cognitive functioning; Schizophrenia

\section{Introduction}

High frequency electromagnetic activity $(>20 \mathrm{~Hz})$, also referred to as the gamma band, has repeatedly been shown to be a correlate of a wide range of cognitive processes, both in animals (e.g. Gray et al., 1989; Singer and Gray, 1995) and in man (Müller et al., 1996; Tallon-Baudry and Bertrand, 1999). An increase in gamma band activity has been observed when 'gestalt' like stimuli (Tallon et al., 1995; Keil et al., 1999) are processed. Moreover, gamma band activity may be modulated by attentional demands (Gruber et al., 1999; Müller, 1998, 2000) and by memory load during the delay phase of a working memory task and may thus index rehearsal processes in memory (TallonBaudry et al., 1998). In language processing, the synchro-

\footnotetext{
* Corresponding author. Fachgruppe Psychologie, Fach D23, Universität Konstanz, 78457 Konstanz, Germany. Tel.: +49-7531-884612; fax: +497531-884601.

E-mail address: johanna.kissler@uni-konstanz.de (J. Kissler).
}

nized firing of cell assemblies at high frequencies has been shown to be sensitive to manipulations of word-class and lexical categories (Pulvermüller, 1999).

In studies investigating the role of high frequency activity in cognitive processes, gamma band is generally induced by the manipulation of physical stimuli. There is a lack of evidence that an increase of gamma activity can be induced by a cognitive task in the absence of external stimulation as would be expected if gamma activity were an ubiquitous index of cognitive processes in humans. Still, in the absence of any kind of external stimulation, coherent firing in the gamma range has been observed. Llinas and colleagues (Llinas and Pare, 1991; Llinas and Ribary, 1993) demonstrated the occurrence of coherent high frequency activity during REM sleep but not in non-REM sleep in humans. Furthermore, they conjectured that such activity may be generally related to information processing and conscious experience (Llinas et al., 1998) and speculated that abnormal states of consciousness and experiences in the absence of external input, such as hallucinations, might be accom- 
panied by gamma band activity gone awry (Llinas et al., 1999).

Indeed, recent research has revealed a possible link between the increase of fast EEG/MEG activity in the brain and altered information processing in schizophrenia. In this illness information processing deficits as well as at times profoundly altered perceptual processes like delusions and hallucinations are well documented, both by cognitive and psychophysiological studies (for a review see Steinhauer et al., 1991). Several older studies had reported increases in fast EEG activity in schizophrenia (Kennard et al., 1955; Itil et al., 1972; Giannitrapani and Kayton, 1974) and while, due to technical limitations, the results of these studies have been debated, in recent years interest in high frequency activity in schizophrenia was rekindled. For instance, Sperling et al. (1996) reported a bilateral increase in magnetoencephalographic activity in the 12.5$30 \mathrm{~Hz}$ range at temporal sites in 3 schizophrenic patients with auditory hallucinations. Furthermore, a study by Baldeweg et al. (1998) describes the case of a patient with intense somatic hallucinations in whom the sources of gamma band electroencephalographic oscillations were mainly localized in somatosensory regions of the right hemisphere. Yet, the incidence of different patterns of gamma band activity in schizophrenia is not well documented, even though several studies report an increase in high beta activity in schizophrenia (Pascual-Marqui et al., 1999; Serafetinides, 1984) or a more anterior localization of beta generators (Dierks et al., 1995) and in spite of the fact that the above mentioned studies indicate that high frequency oscillations may be a correlate of hallucinatory experiences.

Such studies as well as considerations about the general nature of gamma band activity as a neural signature of higher cognitive functioning give momentum to the hypothesis that spectral gamma band activity may reflect some aspect of both normal and erratic information processing. However, this possibility has not been systematically investigated.

Using high resolution MEG, the present study addresses two questions. First, can gamma activity serve as a sensitive index of cognitive processing in a task that is performed purely internally, in the absence of external stimulation? Second, is the amount and topography of gamma activity altered in schizophrenia, possibly reflecting information processing mechanisms characteristic of this illness? If so, this would provide further evidence that gamma band activity is an index of cognitive processing and, moreover, might be a promising neural marker of characteristic forms of information processing in schizophrenia.

Two 5 min periods of spontaneous MEG were recorded from schizophrenia patients and healthy comparison subjects. Subjects were asked to (A) relax with their eyes open and try to rid themselves from any intruding thoughts and (B) perform a mental arithmetic task. A complex task was chosen which poses heavy demands on working memory as subjects have to continuously remember their results while trying to determine the next value to add. This appears particularly suitable for the purpose of the present study since previous research has shown gamma band activity to be sensitive to manipulations of working memory load (Tallon-Baudry et al., 1998) and many studies demonstrated working memory deficits in schizophrenia (e.g. GoldmanRakic, 1994, 1999; Gold et al., 1997; Löw et al., 2000).

\section{Methods}

\subsection{Subjects}

Fifteen DSM IV diagnosed schizophrenia patients (4 females, 11 males; mean age 30.2 years, SD 6.5 years) and 15 healthy controls (mean age 35.8 years, SD 9.4 years) matched for gender and educational status volunteered to participate in this study. All subjects were righthanded as determined by the Edinburgh Handedness Questionnaire (Oldfield, 1971). Participants had no history of neurological disorder. Comparison subjects had no history of psychiatric disease. Patients were inpatients at a state psychiatric hospital and had been diagnosed by a psychiatrist or clinical psychologist. Thirteen of the schizophrenia patients were receiving neuroleptic medication (typical antipsychotics only) at the time of testing. The mean chlorpromazine equivalent was $128.36 \mathrm{mg} /$ day (SD 83.38, min 0.0, $\max 344.60)$. Five of the patients were additionally receiving anticholinergics and one was additionally taking an antidepressant. Patients' clinical symptoms were evaluated on the day of testing by means of the Brief Psychiatric Rating Scale (Overall and Gorham, 1962, as modified by Lukoff et al., 1986) and the PANSS (Kay et al., 1987) and SANS (Andreasen, 1981) symptom scales. Scales were administered by the patients' therapist (clinical psychologist or psychiatrist). The average BRPS score was 40.4 (min 31, $\max 68$ ), the mean PANSS-P score was 14.2 ( $\min 7$, $\max$ 29), PANSS-N was on average $17.93(\min 7$, max 25), mean PANSS-G was $34.2(\min 24$, $\max 50)$ and the SANS scores varied between 3 and 85 (mean 50.33). All participants were informed about the goal of the study and gave written consent prior to the experiment and received a financial bonus for taking part in the experiment.

\subsection{Procedure}

A whole-head magnetoencephalogram was recorded using a 148 channel whole-head magnetometer (Magnes 2500 WH, Biomagnetic Technologies Inc.) while subjects were lying comfortably in a lit, magnetically shielded room. For artifact control, horizontal and vertical electrooculograms and electrocardiograms were co-registered. Prior to measurement participants were familiarized with the recording environment, informed about the tasks and given practice in the arithmetic task. The arithmetic task required assigning to each letter of a nursery rhyme its position in the alphabet as a numerical value and adding 
up the values of all letters consecutively for the $5 \mathrm{~min}$ recording time. The nursery rhyme was familiar to all subjects. Participants were instructed to be as accurate as possible rather than trying to add up the entire rhyme. If a subject's reported sum did not differ by more than $20 \%$ from the actual value at a given position in the rhyme, it was decided that the subject had successfully engaged in the task. Subjects whose reported sum differed by more than $20 \%$ from the actual result were excluded from the experiment. For the resting condition, subjects were instructed not to follow any particular train of thought but to try to relax as much as possible. After each block, participants had to report their calculated result and the position in the rhyme they had reached. They were also interviewed about their strategies and experience. The experimenter obtained a qualitative performance measure by rating participants' performance on a scale from 1 to 10 based on the detail and vividness of their reports. Under both conditions, subjects were instructed to fixate a colored spot attached to the ceiling of the room. Experimental conditions were counterbalanced across subjects.

\subsection{Data analysis}

\subsubsection{Behavioral measures}

The reported sum, the subjects' percentage error and independent performance ratings were recorded as performance measures. Performance differences between groups were assessed using $t$ tests.

\subsubsection{Magnetoencephalographic recording}

MEG data were acquired with a sampling rate of 678.17 $\mathrm{Hz}$ and a band pass filter of 0.1-200 Hz. After reduction of external noise and elimination of low frequency drifts, a correction algorithm implemented in the FOCUS software was applied to eliminate the influence of ocular activity on the MEG. Hereafter, data were visually inspected and the remaining movement, heart beat and muscle artifacts were marked as bad and omitted from further processing. To obtain a stable estimate and to ensure good data quality, only subjects with at least 100 artifact-free epochs were included in the analysis. The preprocessed data were transformed into the frequency domain by means of FOCUS' fast Fourier transform (FFT). A sliding $\cos ^{2}$ FFT window of 1.5 s (1024 sample points) with $50 \%$ overlap was used. The combination of $50 \%$ overlap and multiplication of the window by $\cos ^{2}$ ensures that all time

points contribute equally to the mean power spectrum as $\cos ^{2}(x)+\sin ^{2}(x)=1$. A mean power spectrum was obtained for each subject and condition by averaging across epochs. The resulting mean power spectrum was divided into the following frequency bands: Alpha, 8-12 Hz; Beta1, 13-20 Hz; Beta2, 21-29 Hz; Gamma1, 30-45 Hz; Gamma2, 46-60 Hz (the range from 48 to $50 \mathrm{~Hz}$ was excluded to avoid possible electrical interference); and Gamma3, 61-71 Hz. Due to hardware problems, 8 out of
148 channels had to be excluded from the analysis. For statistical analysis, the remaining 140 channels were grouped into the following 5 channel groups for each hemisphere: frontal, fronto-temporal, posterio-temporal, central and occipital (see Fig. 1).

Overall power differences between tasks and subject group and interactions thereof were analyzed with repeated measures analyses of variance (ANOVA) with the between factor group and the within factors task, hemisphere and site for each frequency band. Interaction effects involving the effect of task on topography were analyzed with repeated measures ANOVAs. For topographic analyses data were normalized according to the procedure suggested by McCarthy and Wood (1985). Power values at each sensor were transformed according to the following rule

$x_{\text {inew }}=\frac{x_{\text {imeas }}-\min }{\max -\min }$

where $x_{\text {inew }}$ is the normalized value at sensor i, $x_{\text {imeas }}$ is the measured value at sensor $i$ and max and min are the maximum and minimum across sensors.

Where appropriate, significance levels are reported with Huynh-Feldt epsilon adjusted degrees of freedom. Post-hoc analyses were performed using Newman-Keuls tests.

\section{Results}

\subsection{Task performance}

The average absolute result reported in the arithmetic task was 173.48 (SD 62.04) for healthy subjects and 144.53 (SD 77.98) for patients. The numerical difference in performance between the groups did not reach statistical significance $(t(28)=1.125, \quad P>0.10)$. Task accuracy was compared as the difference between the reported result and the actual result in percent. This error percentage was $6.6 \%$ (SD 6.48) for controls and $5.61 \%$ (SD 6.53) for patients $(t(28)=1.24, P>0.10)$. Experimenter observed qualitative performance ratings for the arithmetic task were $9.5 \%$ (SD 0.99) for controls and $8.9 \%$ (SD 1.6) for patients $(t(28)=1.55, P>0.10)$. The patients generally received lower ratings for their ability to relax during the resting condition. Comparison subjects received average ratings of $9.2 \%$ (SD 1.27), while the value for patients was $8.27 \%(\mathrm{SD} 1.69)(t(28)=1.94, P=0.06)$.

\section{2. $M E G$ data}

\subsubsection{Effects of task and group on overall MEG power}

In the frequency range between 21 and $29 \mathrm{~Hz}$ (Beta2), patients tended to display more overall magnetic activity than controls $(F(1,28)=3.21, P=0.08)$. Apart from this trend, there were no differences in overall neuromagnetic activity between patients and controls in any of the analyzed frequency bands. Likewise, there were no general effects of task on overall neuromagnetic activity or interactions 


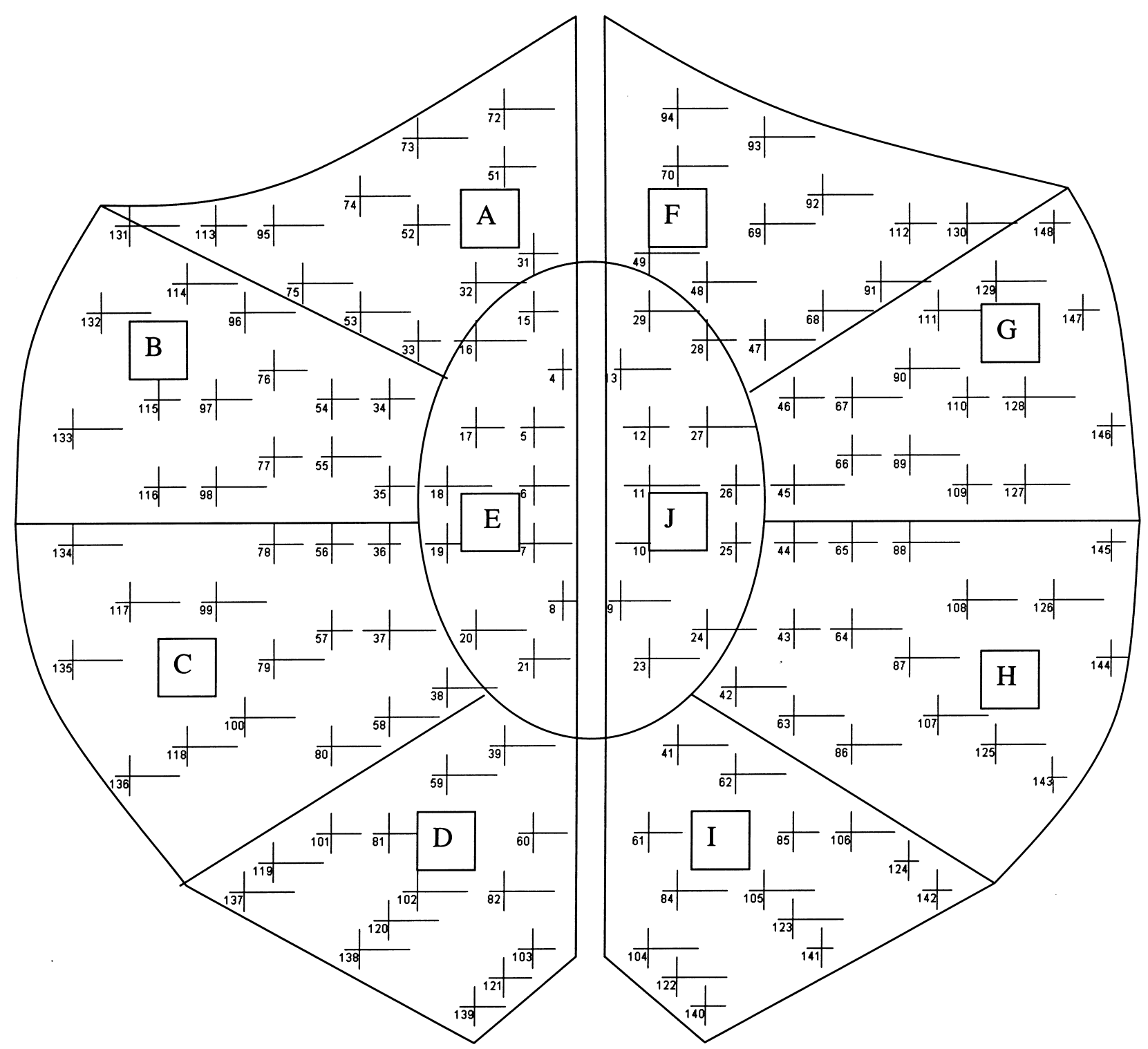

Fig. 1. Schematic illustration of the MEG sensor layout and the 10 channel groups. Channel groups are labeled with capital letters: (A) left frontal; (B) left fronto-temporal; (C) left posterio-temporal; (D) left occipital; (E) left central; (F) right frontal; (G) right fronto-temporal; (H) right posterio-temporal; (I) right occipital; (J) right central.

between task, group and overall neuromagnetic activity in any of the analyzed frequency bands.

\subsubsection{Effects of task and group on scalp distributions}

Table 1 shows how task and group interacted with MEG topography in the analyzed frequency bands. Fig. 2 depicts the power distribution in the gamma range recorded from 5 channel groups in each of the hemispheres separately for healthy subjects (Fig. 2A) and for the schizophrenic patients (Fig. 2B).

In the alpha range, no significant effects of either task or group on MEG topography were observed. Task interacted with recording site in the Beta1 and Beta2 bands $(F(4,112)=3.57, P=0.01$ and $F(4,112)=4.56$,
$P<0.01$, respectively). For both frequency bands, posthoc tests showed that the mental arithmetic led to a significant enhancement in activity at all but central sites $(P<0.05)$.

For Gammal we found a 4 way interaction of task $\times$ hemisphere $\times$ site $\times$ group $\quad(F(4,112)=3.1, \quad P<0.05)$. This interaction was due to a left frontal and left frontotemporal gamma enhancement during mental arithmetic in controls $(P<0.01)$ which was absent in patients.

In the Gamma2 band an interaction of task $\times$ hemisphere $\times$ site $\times$ group was also observed $(F(4,112)=3.22$, $P<0.05$ ), which stemmed from a significant right frontotemporal increase in activity in patients during mental arithmetic $(P<0.05)$, whereas in controls right fronto-temporal 
Table 1

Effects of task and group on MEG topographies in the high frequency bands

\begin{tabular}{|c|c|c|c|}
\hline Frequency band & Effect & $F$ value & $P$ value $^{\mathrm{a}}$ \\
\hline \multirow[t]{4}{*}{ Beta1 } & Group $\times$ site & 0.18 & 0.68 \\
\hline & Task $\times$ site & 3.58 & $0.01 * *$ \\
\hline & Task $\times$ hemisphere $\times$ group & 2.00 & 0.17 \\
\hline & Task $\times$ hemisphere $\times$ site $\times$ group & 0.65 & 0.56 \\
\hline \multirow[t]{4}{*}{ Beta2 } & Group $\times$ site & 1.40 & 0.24 \\
\hline & Task $\times$ site & 4.56 & $0.003 * *$ \\
\hline & Task $\times$ hemisphere $\times$ group & 3.45 & 0.07 \\
\hline & Task $\times$ hemisphere $\times$ site $\times$ group & 2.08 & 0.12 \\
\hline \multirow[t]{4}{*}{ Gamma1 } & Group $\times$ site & 1.5 & 0.21 \\
\hline & Task $\times$ site & 2.01 & 0.12 \\
\hline & Task $\times$ hemisphere $\times$ group & 3.54 & 0.07 \\
\hline & Task $\times$ hemisphere $\times$ site $\times$ group & 3.07 & $0.03 *$ \\
\hline \multirow[t]{4}{*}{ Gamma2 } & Group $\times$ site & 1.97 & 0.12 \\
\hline & Task $\times$ site & 0.55 & 0.64 \\
\hline & Task $\times$ hemisphere $\times$ group & 3.47 & 0.07 \\
\hline & Task $\times$ hemisphere $\times$ site $\times$ group & 3.22 & $0.02 *$ \\
\hline \multirow[t]{4}{*}{ Gamma3 } & Group $\times$ site & 3.00 & $0.03^{*}$ \\
\hline & Task $\times$ site & 2.58 & 0.06 \\
\hline & Task $\times$ hemisphere $\times$ group & 4.82 & $0.04 *$ \\
\hline & Task $\times$ hemisphere $\times$ site $\times$ group & 3.46 & $0.01 * *$ \\
\hline
\end{tabular}

a $* P<0.05, * * P<0.01$.

activity was increased during rest $(P<0.05)$. Moreover, as evident from an interaction of task $\times$ hemisphere $\times$ group $(F(1,28)=0.07, P<0.1)$ there was also a trend for patients to show left hemispheric enhancement during mental arithmetic and right hemispheric enhancement during rest, while the opposite tended to be true for comparison subjects.

In the Gamma3 band topography interacted with group $(F(4,112)=3.00, P<0.05)$. This effect was due to a general activity reduction at fronto-temporal, posteriotemporal and occipital sites in patients $(P<0.05)$. Fig. 3 shows this task-dependent difference in power topography between healthy subjects (top row) and schizophrenia patients (bottom row).

The groups also differed with respect to the task effects on MEG activity in the two hemispheres $(F(1,28)=4.82$, $P<0.05)$. Post-hocs revealed that controls displayed more activity in the right than in the left hemisphere during rest $(P<0.01)$ and greater left than right hemispheric activity during mental arithmetic $(P<0.01)$. Patients, by contrast, displayed no asymmetry at rest and a right hemispheric enhancement during mental arithmetic $(P<0.01)$. The effect of task on the scalp distribution in the two hemispheres differed between the two groups $(F(4,112)=3.5$, $P<0.05)$. Healthy subjects showed a left frontal $(P<0.05)$ and fronto-temporal $(P<0.05)$ enhancement in activity during mental arithmetic, whereas patients showed a right fronto-temporal enhancement during mental arithmetic. This topographic difference is illustrated in Fig. 4. Furthermore, generally task affected the scalp distribution $(F(4,112)=2.5, P<0.1)$, indicating a general widely spread enhancement of activity during mental arithmetic.

In summary, in all frequency bands above $30 \mathrm{~Hz}$, healthy subjects showed a left frontal and fronto-temporal enhancement of activity during mental arithmetic which was either absent or reversed in schizophrenic patients. This pattern is illustrated in Fig. 2 which shows the activity distribution for the 3 gamma bands separately for the two groups. Also, controls exhibited more right than left hemispheric activity at rest, and greater left than right hemispheric activity during mental arithmetic. In patients, by contrast, the enhancement under cognitive activation was more right hemispheric.

\subsection{Medication effects}

Spearman rank correlations were calculated between gamma power at rest and chlorpromazine equivalents in order to determine the effect medication had on gamma activity. The following correlation coefficients were obtained: Gamma1, $\quad r=0.01, \quad P=0.97 ; \quad$ Gamma2, $r=0.02, P=0.99$; Gamma3, $r=-0.04, P=0.87$. None of these reached statistical significance.

\section{Discussion}

The present study served two purposes. On the one hand, we investigated the extent to which gamma band activity can be induced by a purely mental task, i.e. by cognitive activity in the absence of external stimulation. On the other hand, we tested whether such task-dependent activation would differ between healthy subjects and schizophrenia patients. The first issue is important as the assumption that gamma activity can serve as a general indicator of cognitive processing regardless of stimulation has not been explicitly tested. The second issue is of general interest because of considerations about gamma activity as a potential indicator of differential information processing in schizophrenia patients and controls. More specifically, however, the working memory task we used could have been especially indicative as working memory deficits have widely been reported in schizophrenia (Goldman-Rakic, 1994, 1999; Gold et al., 1997; Löw et al., 2000).

With regard to topographic effects of the experimental manipulation, we were clearly able to show that the arithmetic task led to topographical differences in the gamma range. A frontal and fronto-temporal enhancement in gamma power was evident while subjects were performing calculations compared to at rest. This result is consistent with a previous report by Tallon-Baudry et al. (1998) in demonstrating frontal gamma enhancement in a task that draws on working memory. In healthy subjects the mental arithmetic task led to a left lateralized enhancement of gamma activity over left frontal and fronto-temporal sites (see Figs. 2A and 3A). Differences between the report by Tallon-Baudry et al. (1998) and our study are probably due to differences in task and stimulation. In contrast to their study we did not find a significant occipital task effect which could be explained by the absence of visual stimulation in our study. For healthy 

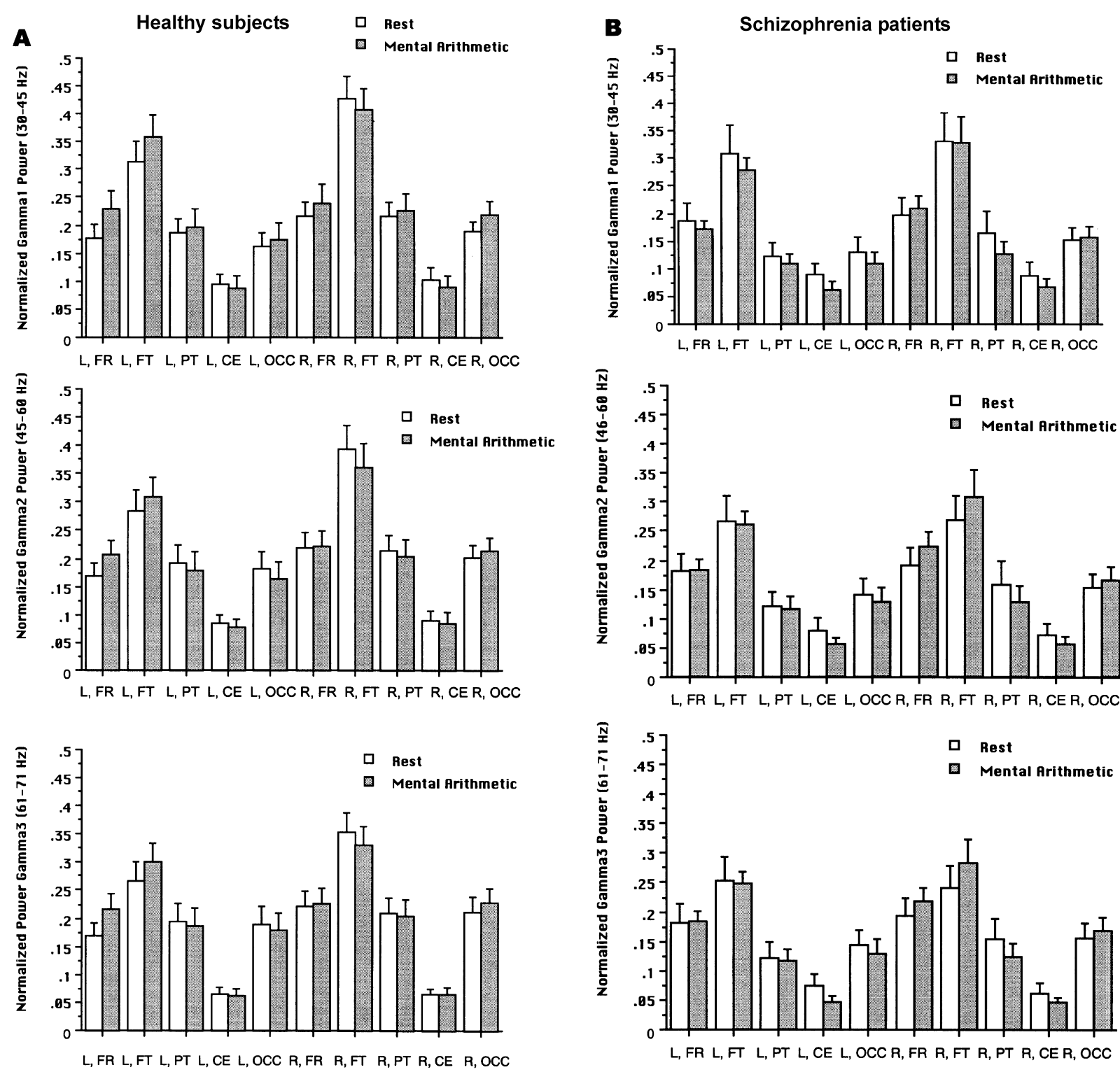

Fig. 2. Bar chart (means and standard errors) of normalized gamma power over different regions of the scalp in controls (A) and patients (B) at rest (open bars) and during mental arithmetic (shaded bars). Regions are abbreviated as follows: L, FR, left frontal; L, FT, left fronto-temporal; L, PT, left posterio-temporal; L, C, left central; L, OC, left occipital; R, FR, right frontal; R, FT, right fronto-temporal; R, PT, right posterio-temporal; R, C, right central; R, OC, right occipital.

subjects the difference in lateralization in frontal areas between their study and ours can be similarly explained, theirs being a visual task whereas we used a task with a strong verbal and covert articulatory component.

The present results concerning the task effect in controls are in line with theoretical considerations about the architecture of working memory (Baddeley, 1986; GoldmanRakic, 1999) and brain imaging studies of working memory (Smith and Jonides, 1999; Ungerleider, 1995). From these studies it has emerged that storage of verbal materials activates Broca's areas and left supplementary and premotor areas, whereas storage of spatial material and object attributes leads to activation in right premotor areas and prefrontal cortex. However, a direct comparison of the results of brain imaging studies and EEG/MEG topography is difficult because of possible localizatory ambiguities underlying such scalp topographies and as the different methodologies measure different aspects of brain activity.

Schizophrenia patients, by contrast, did not show this effect in the Gamma1 frequency range and instead displayed a reversed asymmetry with greater right frontal and frontotemporal activation in the Gamma2 and Gamma3 bands (see Figs. 2B and 4). An absence of left lateralization during working memory activation has been observed in a recent study by Löw et al. (2000), where the contingent negative variation $(\mathrm{CNV})$ was observed to shift towards left frontal sites under working memory challenge in control subjects but not in schizophrenia patients. While these authors interpreted the results as being related to patients' difficulties in activating working memory, our results may suggest an 
$\mid \begin{aligned} & +.14 \\ & -.14\end{aligned}$

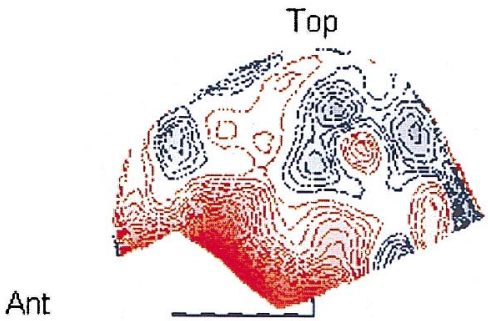

Left Hemisphere

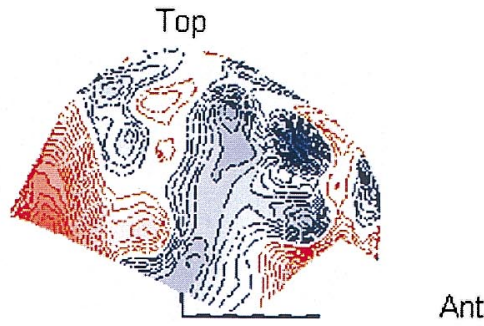

Right Hemisphere

\section{(A): Healthy Subjects}

$\left\{\begin{array}{l}+.14 \\ -.14\end{array}\right.$

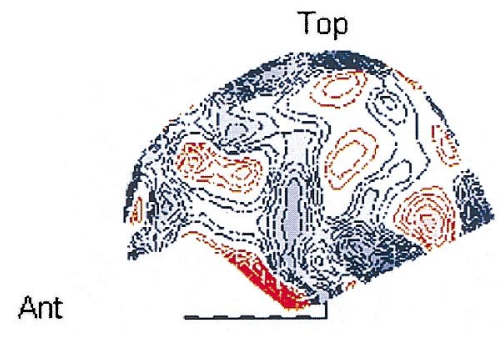

Left Hemisphere

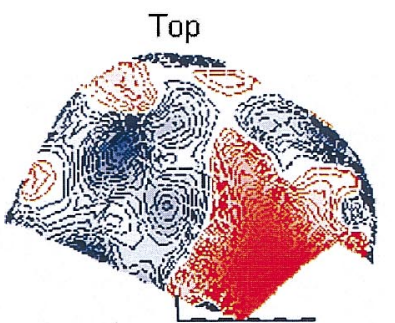

Ant

Right Hemisphere

\section{(B): Schizophrenia Patients}

Fig. 3. Difference map showing normalized gamma power $(30-45 \mathrm{~Hz})$ in the mental arithmetic task minus normalized power at rest for controls (top row) and patients (bottom row). Power values are normalized according to McCarthy and Wood (1985). The red areas indicate an increase in power during cognitive activation.

alternative interpretation: as patients and control subjects did not differ in performance measures we may actually observe a compensatory mechanism as the task we used challenges working memory, yet it is rather complex and non-specific in that it allows subjects to form different stra- tegies. Alternatively, the difference in lateralization while performing the task may fit in with other reports of reduced brain asymmetry in schizophrenia (Crow, 1998; Rockstroh et al., 1998) and represent a trait-like aspect of the illness (Crow, 1997).

$$
\mid \begin{aligned}
& +.4 \\
& -.4
\end{aligned}
$$
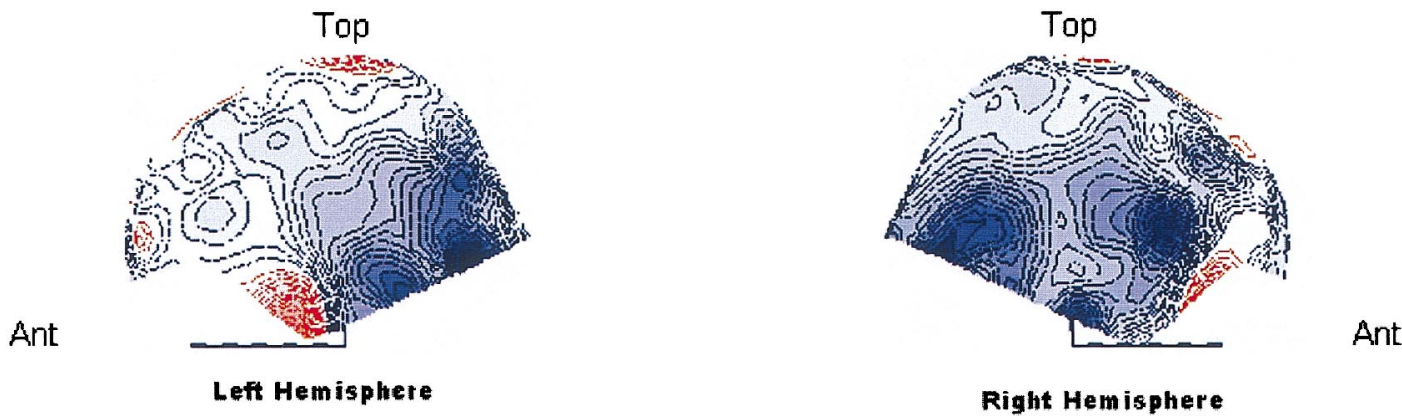

Fig. 4. Difference map showing Gamma3 topography $(61-71 \mathrm{~Hz})$ in healthy comparison subjects minus gamma topography in schizophrenia patients. Power values are transformed according to McCarthy and Wood (1985). The blue areas indicate reduced activity in patients. 
The tendency for patients to display more MEG power in the Beta2 is analogous to findings in previous studies (Kennard et al., 1955; Giannitrapani and Kayton, 1974; Serafetinides, 1984; Dierks et al., 1995; Sperling et al., 1996; Pascual-Marqui et al., 1999). However, in our study this effect was not very strong, suggesting that overall high frequency excess may be more related to the acute, positive symptoms as the patients who participated in the present study were relatively well remitted.

Gamma topography differed between the groups in the highest examined frequency band. Scalp distribution was more central in patients than in controls. Patients had relatively less gamma bilaterally at fronto-temporal, posteriotemporal and occipital sites while there was no difference at frontal and central sites. Previous research indicates the involvement of thalamocortical loops in the generation of gamma activity (Ribary et al., 1991; Llinas and Ribary, 1993) and tentative connections have been established between thalamic bursts and positive symptoms or neuropsychological deficits (Jeanmonod et al., 1996; Llinas and Ribary, 1993) which both occur in schizophrenia. Evidence from molecular research also suggests the involvement of networks of inhibitory neurons in the thalamocortical loop in gamma range firing of pyramidal cells (Whittington et al., 1996). Thus, the present findings appear consistent with other research suggesting abnormalities in the thalamocortical loop leading to disinhibitory phenomena in schizophrenia (Jones, 1997). While the exact mechanisms mediating such abnormalities are unclear at present, abnormalities in thalamocortical oscillations may well underlie our present findings and fit into an overall picture of aberrant inhibitory activity in schizophrenia.

The effects of neuroleptic medication are a source of concern in most electrophysiological studies of schizophrenia. Such a concern may have been heightened in the present study because of recent reports that patients treated with the atypical antipsychotic clozapine had significantly elevated levels of high frequency activity in comparison to patients treated with haloperidol (Sperling et al., 1999), which could have been either caused by the medication or due to some other illness-related process more prevalent in the clozapine than in the haloperidol group. In our study none of the patients were receiving atypical antipsychotics and two were without medication. Also, chlorpromazine equivalents did not correlate with the amount of gamma. Even though some of our patients were additionally receiving anticholinergics and the effect of interactions of neuroleptics with anticholinergics is largely unknown and may complicate interpretations, this suggests that different patterns of gamma activity can arise in schizophrenia independently of neuroleptic medication.

The possibility of artifact contamination is another ever present issue in studies addressing the functional significance of high frequency brain activity. While this possibility can hardly be completely ruled out, the patterns of results can be used to argue against such contamination. We observe the strongest task effects at left frontal and frontotemporal sites in controls and at right frontal and frontotemporal sites in patients. Both the location and the lateralization of the effect make an artifact contamination less likely as high frequency artifacts are more commonly observed in temporal and occipital regions and more so in patients. In the present study, however, patients displayed relatively less gamma at temporal and occipital sites. Moreover, group-specific differences in lateralization under cognitive demands would appear unlikely if effects were artifactual.

In summary, we successfully demonstrated that gamma band activity can be enhanced by a cognitive task without external stimulation and thus can reflect aspects of cognitive functioning both in healthy people and in schizophrenia patients. Both groups showed a frontal and fronto-temporal increase in high frequency MEG activity which, however, was left lateralized in controls but lateralized to the right in patients. High frequency activity also tended to be increased in schizophrenia patients and the topographies of this activity differed between the two groups, suggesting that abnormalities in the thalamocortical loop may be responsible for some features of the illness.

\section{Acknowledgements}

We thank Drs K. Pröpster and H. Watzl for providing the diagnostics and information on the clinical status of the patients. Thanks are also extended to Jennifer McDowell for helpful comments on a previous version of the manuscript. This research was supported by the Deutsche Forschungsgemeinschaft.

\section{References}

Andreasen N. Scale for the assessment of negative symptoms: SANS, Iowa City, IA: University of Iowa, 1981.

Baddeley A. Working memory, London: Oxford University Press, 1986.

Baldeweg T, Spence S, Gruzelier J. Gamma band electroencephalographic oscillations in a patient with somatic hallucinations. Lancet 1998;352:620-621.

Crow TJ. Is schizophrenia the price that Homo sapiens pays for language? Schizophr Res 1997;28(2):127-141.

Crow TJ. Schizophrenia as a transcallosal misconnection syndrome. Schizophr Res 1998;30(2):111-114.

Dierks T, Strik WK, Maurer K. Electrical brain activity in schizophrenia described by equivalent dipoles of FFT-data. Schizophr Res 1995;14:145-154.

Giannitrapani D, Kayton L. Schizophrenia and EEG spectral analysis. Electroenceph clin Neurophysiol 1974;36:377-386.

Gold JM, Carpenter C, Randolph C, Goldberg TE, Weinberger DR. Auditory working memory and Wisconsin Card Sorting Test performance in schizophrenia. Arch Gen Psychiatry 1997;54:159-165.

Goldman-Rakic PS. Working memory dysfunction in schizophrenia. J Neuropsychiatry Clin Neurosci 1994;6:348-357.

Goldman-Rakic PS. The physiological approach: functional architecture of working memory and disordered cognition in schizophrenia. Biol Psychiatry 1999;46(5):650-661.

Gray CM, König P, Singer W. Oscillatory responses in cat visual cortex 
exhibit inter-columnar synchronization which reflects global stimulus properties. Nature 1989;338:334-337.

Gruber T, Müller MM, Keil A, Elbert T. Selective visual-spatial attention alters induced gamma band responses in the human EEG. Clin Neurophysiol 1999;110:1-12.

Itil TM, Saletu B, Davis S. EEG findings in chronic schizophrenics based on digital computer period analysis and analog power spectra. Biol Psychiatry 1972;5:1-13.

Jeanmonod D, Magnin M, Morel A. Low-threshold calcium spike bursts in the human thalamus. Common physiopathology for sensory, motor and limbic positive symptoms. Brain 1996;119:363-375.

Jones EG. Cortical development and thalamic pathology in schizophrenia. Schizophr Bull 1997;23(3):483-501.

Kay S, Fiszbein A, Opler LA. The positive and negative syndrome scale (PANSS) for schizophrenia. Schizophr Bull 1987;13:261-276.

Keil A, Müller MM, Ray W, Gruber T, Elbert T. Human gamma band activity and perception of a gestalt. J Neurosci 1999;19(16):7152-7161.

Kennard MA, Rabinovitch MS, Fister WP. The use of frequency analysis in the interpretation of the EEGs of patients with psychological disorders. Electroenceph clin Neurophysiol 1955;7:29-38.

Llinas R, Pare D. Of dreaming and wakefulness. Neuroscience 1991;44:521-535.

Llinas R, Ribary U. Coherent 40-Hz oscillation characterizes dream state in humans. Proc Natl Acad Sci USA 1993;90:2078-2081.

Llinas R, Ribary U, Contreras D, Pedroarena C. The neuronal basis for consciousness. Phil Trans R Soc Ser B 1998;353:1841-1849.

Llinas RR, Ribary U, Jeanmonod D, Kronberg E, Mitra PP. Thalamocortical dysrhythmia: a neurological and neuropsychiatric syndrome characterized by magnetoencephalography. Proc Natl Acad Sci USA 1999;96:1522-1527.

Löw A, Rockstroh B, Harsch S, Berg P, Cohen R. Event-related potentials in a working-memory task in schizophrenics and controls. Schizophr Res 2000 in press.

Lukoff D, Liberman RP, Nuechterlein KH. Symptom monitoring in the rehabilitation of schizophrenic patients. Schizophr Bull 1986;12(4):578-602.

McCarthy G, Wood CC. Scalp distributions of event-related potentials: an ambiguity associated with analysis of variance models. Electroenceph clin Neurophysiol 1985;62:203-208.

Müller MM. Hochfrequente oszillatorische Aktivitäten im menschlichen Gehirn (High frequency oscillatory neural activities in the human brain). Zeitschrift für Experimentelle Psychologie 2000 in press.

Müller MM, Bosch J, Elbert T, Kreiter A, Sosa MV, Sosa PV, Rockstroh B. Visually induced gamma-band responses in human electroencephalographic activity - a link to animal studies. Exp Brain Res 1996;112: 96-112.

Müller MM, Teder-Salejarvi W, Hillyard SA. The time course of cortical facilitation during cued shifts of spatial attention. Nat Neurosci 1998;1(7):631-634.

Oldfield RC. Assessment and analysis of handedness: the Edinburgh inventory. Neuropsychologia 1971;9:97-113.

Overall E, Gorham DR. The Brief Psychiatric Rating Scale. Psychol Rep 1962;10:799-812.

Pascual-Marqui R, Lehmann D, Koenig T, Kochi K, Merlo MCG, Hell D, Koukkou M. Low resolution brain electromagnetic tomography (LORETA) functional imaging in acute, neuroleptic-naive, firstepisode, productive schizophrenia. Psychiatry Res: Neuroimaging 1999;90:169-179.

Pulvermüller F. Words in the brain's language. Behav Brain Sci 1999;22:253-336.

Ribary U, Ioannides AA, Singh KD, Hasson R, Bolton JP, Lado F, Mogilner A, Llinas R. Magnetic field tomography of coherent thalamocortical 40$\mathrm{Hz}$ oscillations in humans. Proc Natl Acad Sci USA 1991;88(24):11037-11041.

Rockstroh B, Clementz BA, Pantev C, Blumenfeld LD, Sterr A, Elbert T. Failure of dominant left-hemispheric activation to right-ear stimulation. NeuroReport 1998;9(17):3819-3822.

Serafetinides EA. EEG lateral asymmetries in psychiatric disorders. Biol Psychol 1984;19(3-4):237-246.

Singer W, Gray CM. Visual feature integration and the temporal correlation hypothesis. Annu Rev Neurosci 1995;18:555-586.

Smith EE, Jonides J. Storage and executive processes in the frontal lobes. Science 1999;283:1657-1661.

Sperling W, Möller M, Kober H, Vieth J, Barocka A. Spontaneous slow and fast MEG activity in schizophrenics with auditory hallucinations. Neurol Psychiatry Brain Res 1996;4:225-230.

Sperling W, Vieth J, Martus M, Demling J, Barocka A. Spontaneous slow and fast MEG activity in male schizophrenics treated with clozapine. Psychopharmacol (Berl) 1999;142(4):375-382.

Steinhauer SR, Gruzelier JH, Zubin J, editors. Neuropsychology, psychophysiology and information processing. Handbook of schizophrenia, vol. 5. Amsterdam: Elsevier, 1991

Tallon C, Bertrand O, Bouchet P, Pernier J. Gamma range activity evoked by coherent visual stimuli in humans. Eur J Neurosci 1995;7(6):12851291.

Tallon-Baudry C, Bertrand O. Oscillatory gamma activity in humans and its role in object representation. TICS 1999;3:151-162.

Tallon-Baudry C, Bertrand O, Perronet F, Pernier J. Induced gamma band activity during the delay of a visual short-term memory task in humans. J Neurosci 1998;18(11):4244-4254.

Ungerleider LG. Functional brain imaging studies of cortical mechanisms for memory. Science 1995;270:769-775.

Whittington M, Traub RD, Jeffreys JRD. Synchronized oscillations in interneuron networks driven by metabotropic glutamate receptor activation. Nature 1996;373:612-614. 